\title{
Erratum
}

\section{Function Weighted Measures and Orthogonal Polynomials on Julia Sets ${ }^{1}$}

D. Bessis, J. S. Geronimo, and P. Moussa

Theorem 3.4 in the above paper is not quite strong enough to give Corollary 3.1. However, the following strengthening of Theorem 3.4 does imply Corollary 3.1. (Note that $z \notin J$ in the hypothesis of Corollary 3.1 should read $z \notin I$.)

Theorem 3.5. Let $c, \Delta, \gamma$, and $\varphi$ be as in (3.20) and (3.21) in the above paper. Let

$$
f=\max _{s}\left\|p_{s}\right\|_{\infty}, \quad s=0,1,2, \ldots, d^{2}-1 \text {. }
$$

Then

(*) $\quad\left\|p_{m d^{n}+s}\right\|_{\infty} \leq f \varphi^{n-1}, \quad n=1,2, \ldots, \quad s=0,1, \ldots, d^{n}, \quad m=1,2, \ldots, d-1$.

Proof. We prove the result by induction since $\left\|p_{d^{2}}\right\|_{\infty}=\left\|p_{d}(T(x))\right\|_{\infty}=\left\|p_{d}(x)\right\|_{\infty}$ the result is true for $n=1$. Assume now that $(*)$ is true for $n=k-1$. If $s=l d^{i}$, $l<d$, and $0<i \leq k$, then

$$
\begin{aligned}
\left\|p_{m d^{k}+s}\right\|_{\infty} & =\left\|p_{m d^{k}+l d^{\prime}}\right\|_{\infty} \\
& =\left\|p_{m d^{k-1}+l d^{i-1}}(T(x))\right\|_{\infty} \\
& =\left\|p_{m d^{k-1}+l d^{i-1}}(x)\right\|_{\infty} \leq f \varphi^{k-2} \leq f \varphi^{k-1}
\end{aligned}
$$

since $\varphi \geq 1$. Write $s=l d^{i}+j, 0<i<k, j=1,2, \ldots, d-1$, then from (3.18), (3.21), and (3.23) of the above paper we find

$$
\begin{aligned}
\left\|p_{d\left(m d^{k-1}+l d^{i-1}\right)+j}\right\|_{\infty} & \leq \frac{c}{\gamma}\left(\Delta\left\|p_{m d^{k-1}+l d^{i-1}}\right\|_{\infty}+\left\|p_{m d^{k-1}+l d^{i-1}+1}\right\|_{\infty}\right) \\
& \leq \frac{c}{\gamma}(\Delta+1) f \varphi^{k-2}=f \varphi^{k-1}
\end{aligned}
$$

which gives the result.

Date received: October 25, 1989. Communicated by Mourad Ismail.

${ }^{1}$ D. Bessis, J. S. Geronimo, and P. Moussa (1988): Function weighted measures and orthogonal polynomials on Julia sets. Constr. Approx., 4:157-173. 
Remark. Using the fact that $\prod_{i=1}^{n} a(n d+i)=a(n+1)$ and (3.18) modified so that it works for the orthonormal polynomials, i.e.,

$$
\hat{p}_{n d+j}=\frac{1}{w(x)}\left\{\frac{a(n+1)}{a(n d+j+1)} \hat{p}_{d-j-1}^{(n d+j+1)}(x) \hat{p}_{n d}(x)+\frac{a(n+1)}{a(n d+1)} \hat{p}_{d-1}^{(n d+1)}(x) \hat{p}_{(n+1) d}(x)\right\}
$$

and using techniques similar to those above it is not hard to show that the orthonormal polynomials exhibit regular asymptotic behavior [1].

\section{Reference}

1. H. STAHL, V. TOTIK (1990): Nth root asymptotic behavior of orthonormal polynomials. In: Orthogonal Polynomials: Theory and Practice (P. Nevai, ed.). NATO ASI Series. Dordrecht: Kuwer, pp. 395-417. 